\title{
Innovative Experimental Platform Design and Teaching Application of the Internet of Things
}

\author{
http://dx.doi.org/10.3991/ijoe.v11i6.5025 \\ Cai Ken ${ }^{1}$, Tie Fenglian ${ }^{1}$, Huang Haoran ${ }^{1}$, Lin Hanhui ${ }^{2}$ and Chen Huazhou ${ }^{3, *}$ \\ ${ }^{1}$ Zhongkai University of Agriculture and Engineering, Guangdong, China \\ ${ }^{2}$ Guangdong University of Finance and Economics, Guangzhou, China \\ ${ }^{3}$ Guilin University of Technology, Guilin, China
}

\begin{abstract}
By analyzing the current situation of experimental platforms based on the Internet of Things (IOT) and the cultivation of talents, we established the talents' cultivating orientation and an experiment platform for innovation. In accordance with the requirements of a practical and creative curriculum, a method of modularization was adopted to design a platform. With this, the platform can basically satisfy the needs of varying teaching experiments, which can increase the opportunities for students' comprehensive application. The platform has been widely used in experimental and practice teaching in the past three years, such as in synthetic experiments, graduate programs, and practice projects at Zhongkai University of Agriculture and Engineering, Guilin University of Technology, etc. Results show that the innovative experimental platform has broad application prospects and effectiveness to meet the requirements of students' in-depth learning and research of IOT technologies. The platform expanded the students' basic understanding of IOT and improved their innovation.
\end{abstract}

Index Terms-Internet of Things, educational platform, innovation ability, higher education

\section{INTRODUCTION}

The Internet of Things (IOT), the network through which data can be interacted with and communicated between things, is extending itself to users on the basis of the Internet. IOT is the fourth tremendous transformation (succeeding the computer, Internet, and mobile communication) in the era of global informationalization. It has accelerated the real-time Internet of entities featuring object perception and information worldwide and has realized interconnections in a wide scope. The IOT industry has been playing a strategic role in China's IT industry and has attracted increasing attention. IOT, which has expanded the employment of talents, is integrated with multiple disciplines that cover basic technologies, including built-in, radio frequency identification, sensor networks, measurement and control, wireless data communication, and other relevant fields such as software development and computer science. IOT is emerging with strong momentum. China is pushing IOT development because of its significant role in accelerating the newly developing strategic industry and, thus, stimulating the national economy. We are required to cultivate the talents in IOT, improve ability in IOT application and design, and enhance innovative ability to fully seize the IOT economy as the new economic growth point in this age. IOT talents are required to master not only the technology of sensors, microprocessors, built-in technology, and relevant software but also the latest technology of wireless communication, high-frequency designs, low-power wireless sensor networks, and $3 \mathrm{G}$ wireless network designs. To achieve this goal, IOT teaching must be closely related to experimental teaching. Establishing a technological and innovative platform can aid in the training and innovative thinking development of students; such a platform can also help students transform knowledge to practice and create breakthroughs. Popular overseas IOT experimental platforms include Kansei[1], WISEBED[2], FlockLab[3], SmartSantander[4], CONET-IT[5], DES-Testbed[6], INDRIYA[7], W-iLAB.t[8], MOTEL[9], TWIST[10].These platforms have a wide and complicated scope, a high price, and a large quantity of maintenance work. In China, Du et al. [11] designed a comprehensive training platform based on perception, transport, and application layers; Zhou et al. [12] presented a set of experimental systems of IOT based on Zigbee and MEMS. Gesture measuring experiments, target localization and tracking experiments, and intelligent control experiments can be designed with the system. Lu Shaoping et al. [13] studied a logistics management-oriented IOT teaching experimentation platform with seven subsystems, including modules of production, supermarket, home-stay, and logistics. The educational platform of IOT used in high schools and universities in the current phase is still at the exploration stage. The type of platform used plays an important role in IOT education. Therefore, determining how to construct a platform with desirable characteristics and how to use it to improve the teaching process are indispensible.

\section{DEMAND ANALYSIS}

\section{A. Current situation of professional talent cultivation in the IOT}

In September 2009, Beijing University of Posts and Telecommunications and Wuxi City signed an agreement to cooperatively expand the technology research and industrial development of sensor networks. The cooperation focused on sensor networks involving optical communications, wireless communications, computer control, multimedia, network, software, electronics, automation, and other technologies. The first national research institute of IOT was established in Nanjing University of Posts and Telecommunications on September 10, 2009, which represented the beginning of IOT products. The first national School of Engineering of IOT was established at Jiangnan University on June 10, 2010. In 2011, the Ministry of 
Education gave approval for 37 universities to offer majors relevant to IOT Engineering.

\section{B. Training objectives of IOT major}

Instead of a single technology, a wide range of technologies are integrated into an IOT. IOT talents should not only master the basic knowledge of math, science, economics, management science, and engineering science required by the major but also acquire the basic knowledge skills and methods of computer, communication, electronics, and sensing relevant to the IOT. Aside from possessing excellent professional ability and foreign language ability, talents should also be capable of researching IOT technologies or planning, analysis, design, implementation, operation, and maintenance of the IOT system. Thus, an IOT technology major is a typical interdisciplinary program that involves a series of core technologies, such as communications, sensors, networks, and RFID intelligent identification. Knowledge of the different disciplines should be integrated and assimilated effectively through creative practical teaching based on projects.

\section{Urgency of building experimental platform of IOT}

Despite being an emerging technology, IOT can greatly increase economic benefits and reduce production costs to hasten the recovery of the global economy. Striving for the advantages of IOT, powerful nations in traditional information fields (such as the United States, Japan, South Korea, and Europe) have invested heavily in IOT research. China has attached importance to IOT research by launching a series of preferential policies and incentives. However, advanced key technologies are crucial to maintain the sustainable and rapid growth of IOT. Training of talents is one way of staying ahead of the others. Current IOT students gain knowledge mainly from the teachers' lectures. Such a learning method cannot provide practical operation experiences. Hence, students cannot deeply analyze and digest the IOT theories; these students also lack innovative and exploration abilities. Therefore, colleges should establish experimental platforms of IOT to help students easily adapt to actual working environments after graduation. An innovated experimental platform of IOT has been established in this project.

\section{Principle in building an experimental platform of the IOT}

A platform of IOT should be designed to support various business needs, including the common and personalized needs of different businesses. In addition, the platform design should be simple and easy to use with a friendly and uniform system interface. Only a platform meeting the above standards can be highly practical and operational. Users should be able to navigate all of the services provided by the platform after simple training. Moreover, standardized protocols and technology should be adapted to the platform design of the IOT to ensure that the platform design is based on certain specifications and meets standards in terms of port, data format, and communication protocol. Eventually, the platform can satisfy the demands of real-time data query, simple deployment, easy extension, and quick start. Finally, possible issues related to increasing IOT access to sensors include the rapid increase of data collection and the expansion of the collection scope. Software and hardware for platform optimization are needed to solve property bottlenecks in the platform during operation; thus, the platform needs full extendibility. The platform also needs favorable feature access solutions to meet the growing and changing demands. Therefore, the platform should follow the principles of a service-oriented system, ensuring the system has convenient means of adding, deleting, or modifying the functions of each module. Furthermore, the platform must adapt to changes in business needs while not influencing the normal operation of the main body of the platform.

\section{FeAtures Of The Platform}

The platform is designed to help students acquire the related knowledge of IOT and have a deep understanding of the basic principles and theories, such as the protocols. After launching experiments using the platform, the students are expected to perform IOT management, application, and design as well as redevelopment to become future talents in the IOT industry. Based on the above analysis, this research has developed a new type of educational platform for IOT application with the following features.

1) Most of the existing IOT platforms use only one form of wireless technological standard, such as Zigbee network technology, to collect and process the information at the sensor nodes. This experimental platform has an updated core gateway to integrate heterogeneous wireless network technologies, including Zigbee network, Bluetooth network, 2.4G wireless module network, and WiFi wireless monitoring network. Data can transport without any obstacles in the entire platform.

2) With the change of the application environment, corresponding network technologies are provided for collection data to be transmitted and processed.

3) Aside from common sensors such as temperature, humidity, and pressure, the platform is equipped with different sensors and some reserved interfaces for extension. GPS modules can help determine the longitude and latitude of the sensors. In addition, the platform can be connected to a mini-network camera and utilize a WiFi network to collect video and visual information and transmit the core gateway to process.

4) The IOT gateway analyzes the data collected by the sensors. The gateway transmits an instruction to start corresponding devices at the network nodes and operate irrigation, atomization, and heating as well as unlock the camera monitor in the presence of abnormal data. The WiFi network can be used to control the gateway with a smart handheld device.

5) The platform has a wide range of applications and expandability. The platform can support sufficient experiments to help master basic IOT knowledge. It can also support several extensible experiments, such as configuration of wireless sensor networks, location of sensor nodes without GPS modules, optimization of the wireless network energy, and sensor and communication network integration.

\section{Core Hardware Design}

This platform includes three components, namely the IOT gateway module, network monitoring module, and operating device. As shown in Fig. 1, the IOT gateway is the core module. It is based on the embedded processing system, and multiple units such as USB, WiFi network card, and serial ports are integrated. 


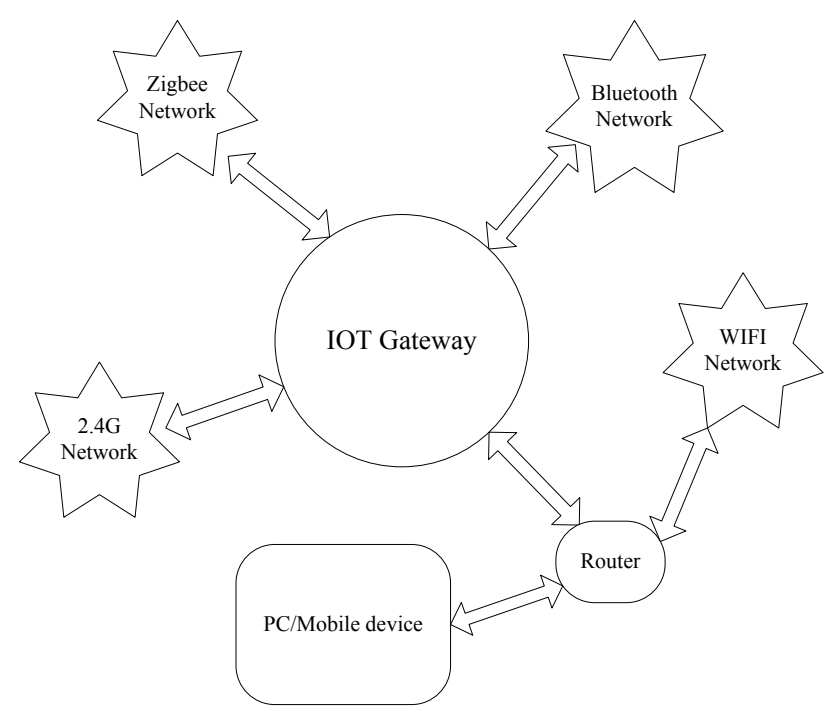

Figure 1. Architecture of IOT gateway in the experimental platform

\section{A. IOT gateway design}

The designed platform adopts various wireless network technology standards, including Zigbee, Bluetooth 4.0, $2.4 \mathrm{GHz}$, and $\mathrm{WiFi}$. These technologies have the same frequency band but different communication protocols. Therefore, the key point is to design the core gateway by integrating different network protocols to control the entire system with a computer or handheld smart devices.

\section{B. Design of integrated serial ports in various forms}

The data collected by the Zigbee network, the Bluetooth network, or the $2.4 \mathrm{G}$ wireless network are transmitted to sink nodes and stored in registers. The core gateway sends a request to every sink node at a certain cycle. Data stored in the requested node are transmitted to the gateway. The IOT gateway verifies the received data and writes the verified data to the corresponding addresses in the database. Users can check the data servers through a WiFi network using a PC or portable equipment to obtain the data at each node.

In this design, the IOT gateway is configured with only one USB port. A USB concentrator is used to connect multiple sink nodes. Time division multiplexing is applied to realize several-to-one data transmission. In specific, different communication networks transmit data to the gateway. As shown in Fig. 2, the server sends a data request and instructions for control to the sink node in the Zigbee network and waits for an answer. When the Zigbee network sends the data back in time, the gateway verifies and saves the data; otherwise, the gateway terminates it at a designated time. The process is the same with the $2.4 \mathrm{G}$ and Bluetooth networks and returns it to the Zigbee network.

This design has only one USB port at each IOT gateway design. A USB concentrator has been adopted to connect the equipment at multiple rendezvous points in the network, allowing data transmission via multiple equipment through one port after another. Time division multiplexing is applied, allowing data transmission through only one communication network to the gateway. If the transmission is successful, then it is continued; oth-

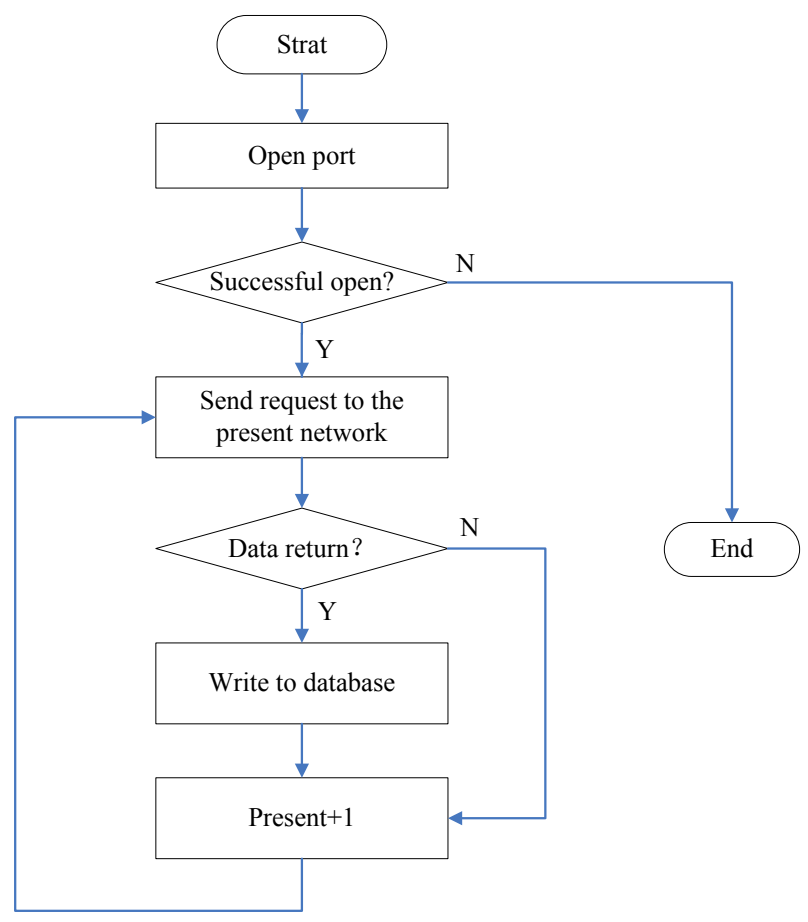

Figure 2. IOT gateway data transmission control

erwise, the transmission is terminated at a certain range of time and the next transmission is performed.

\section{EXPERIMENTAL PLATFORM APPLICATION}

The platform has been widely used in the following four aspects since its introduction in teaching three years ago. It has also been implemented in more than a dozen colleges and universities for teaching experiments: Zhongkai University of Agriculture and Engineering, Guilin University of Technology, Guangdong University of Finance and Economics, South China University of Technology, Wuyi University, South China Agricultural University, and Xinyu University.

\section{1) Completion of the basic IOT project}

The educational experiment platform serves various functions. For instance, it can conduct data acquisition at the sensor nodes with Zigbee/Bluetooth/2.4GHz modes, self-organizing network sensors, sensor data exploration, and control and video monitoring. Adopted by computer science, networking, electronics and information engineering, communication engineering, and other professional majors, the platform can help students understand and master knowledge of IOT.

More than 1,200 students have experimented with the platform in the past three years, but the total service data include more than 10,000 individuals.

2) Innovative experiment and comprehensive training

The educational experiment platform has provided opportunities for teachers and students to improve their innovative experiments and comprehensive training. Associated with actual needs in life and production, the IOT networking application has been formulated on the basis of the platform. This platform can establish an IOT system for home-stay, transportation, mining, aquaculture, and other areas. Fig. 3 shows an agricultural environmental monitoring and management system that was established based on this IOT platform. 


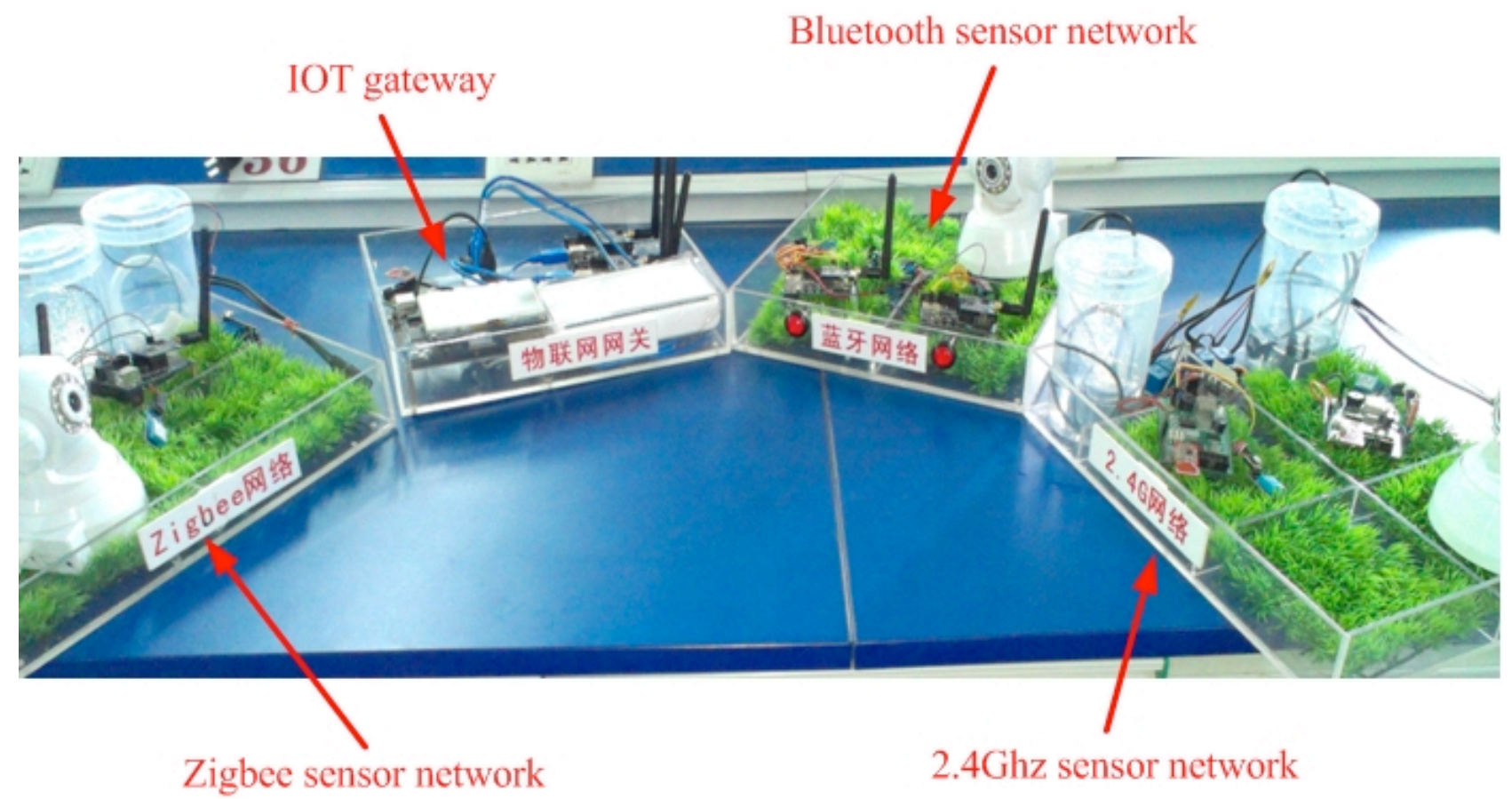

Figure 3. Experimental platform based on IOT

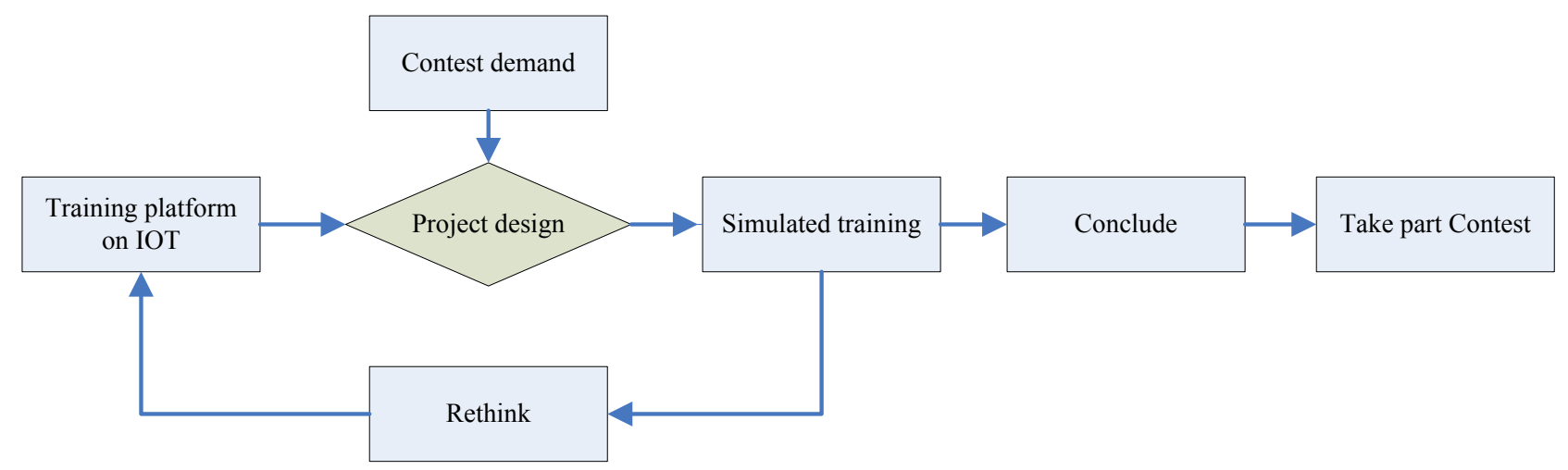

Figure 4. Training process

The management system adopts the Zigbee network, Bluetooth, and $2.4 \mathrm{GHz}$ to monitor agricultural farmland, warehouses, and greenhouses and to collect environmental data. The collected data are integrated at the IOT gateway and saved in the corresponding database. Users can check the servers through a WiFi network using a PC or portable equipment to obtain the data at each node. They can also manually control the braking devices at each network node to operate irrigation, air moistening, or automatic mode settings. If the data at the nodes exceed the preset value, the automatic modes perform irrigation and air humidity.

3) Assistance to student training in the improvement of competitiveness

Instructors can use this platform to perform simulated training for students to improve their competitiveness in multiple electronic contests. With training on this platform, the students can find and solve problems and carry on further training by adjusting parameters and updating schemes to increase their competitiveness. The entire process is shown in Fig 4. With the help of the IOT-based platform applied in practice, the contest participators have been granted 21 provincial awards in the past three years.

4) Assistance to the graduation design course

A total of 116 students have been instructed and guided in their graduation design course, and more than 37 students have carried out research and development on this platform in relation to their theses in the 3 years since the inception of this platform.

\section{CONCLUSION}

The IOT educational platform applied to higher school education is presently under research. This paper describes the design and implementation of a new IOT experimental platform. This platform uses a built-in operational system in the design of an IOT gateway and integrates at least three wireless network technologies. It can assist students in their understanding of the basic framework and hardware components of IOT and help them conduct innovative practice. This platform will be further developed and improved in accordance with updated IOTrelated technologies. 


\section{REFERENCES}

[1] A. Arora, E. Ertin, R. Ramnath, M. Nesterenko, and W. Leal, "Kansei: a high-fidelity sensing testbed," IEEE Internet Computing, vol.10, no.2, pp. 35-47, 2006. http://dx.doi.org/10.1109/ MIC. 2006.37

[2] G. Coulson, B. Porter, I. Chatzigiannakis, C. Koninis, S. Fischer, D. Pfisterer, D. Bimschas, T. Braun, P. Hurni, M. Anwander, G. Wagenknecht, S. Fekete, A. Kröller, and T. Baumgartner, "Flexible experimentation in wireless sensor networks," Communications of the ACM, vol.55, no.1, pp. 82-90, 2012. http://dx.doi.org/10.1145/2063176.2063198

[3] R. Lim, F. Ferrari, M. Zimmerling, C. Walser, P. Sommer, and J. Beutel, "Flocklab: a testbed for distributed, synchronized tracing and profiling of wireless embedded systems," in: Proc. of International Conference on Information Processing in Sensor Networks, Philadelphia, Pennsylvania, USA, 2013. http://dx.doi.org/10.1145/ 2461381.2461402

[4] M. Nati, A. Gluhak, H. Abangar, and W. Headley, "Smartcampus: a usercentric testbed for internet of things experimentation," in: Proc. of International Symposium on Wireless Personal Multimedia Communications, Atlantic City, NJ, USA, 2013.

[5] A. Jimenez-Gonzalez, J.R.M.-D. Dios, and A. Ollero, “An integrated testbed for cooperative perception with heterogeneous mobile and static sensors," Sensors, vol.11, no.12, pp. 11516-11543, 2011. http://dx.doi.org/10.3390/s111211516

[6] O. Hahm, M Güneş, and K. Schleiser, "Des-testbed a wireless multi-hop network testbed for future mobile networks," in: Proc. of GI/ITG KuVS Workshop on Future Internet, Hannover, Germany, 2010.

[7] M. Doddavenkatappa, M. Chan, and A. Ananda, "Indriya: a lowcost, 3d wireless sensor network testbed," in: Testbeds and Research Infrastructure. Development of Networks and Communities, Thessanoliki, Greece, 2012. http://dx.doi.org/10.1007/978-3642-29273-6_23

[8] S. Bouckaert, W. Vandenberghe, B. Jooris, I. Moerman, and P. Demeester, "The W-iLAB.t testbed," in: Testbeds and Research Infrastructures. Development of Networks and Communities, Shanghai, China, 2011. http://dx.doi.org/10.1007/978-3-64217851-1 11

[9] E. Förster, A. Förster, T. Leidi, K. Garg, D. Puccinelli, F. Ducatelle, S. Giordano, and L.M. Gambardella, "Poster abstract: motel: towards flexible mobile wireless sensor network testbeds," in: Proc. of the 8th European Conference on Wireless Sensor Networks, Bonn, Germany, 2011.

[10] V. Handziski, A. Köpke, A. Willig, and A. Wolisz, "TWIST: a scalable and reconfigurable testbed for wireless indoor experiments with sensor networks," in: Proc. Workshop on Multi-hop Ad Hoc Networks: From Theory to Reality, Florence, Italy, 2006. http://dx.doi.org/10.1145/1132983.1132995
[11] W. Du and J. Pan, "Design of comprehensive training platform of Internet of things," Experimental Technology and Management, vol.39, no.9, pp. 63-67, 2013.

[12] L. Zhou, H. Wu, and S. Lin, "Research on comprehensive experiments of Internet of things based on Zigbee and MEMS," Experimental Technology and management, vol.35, no.5, pp. 203-218, 2014.

[13] S. Lu, Y. Gan, J. Song, and J. Wang, "Study on Logistics Management Specialty Oriented IOT Teaching Experimentation Platform," Logistics Technology, vol.33, no.11, pp. 462-467, 2014.

\section{AUTHORS}

Ken Cai is with the School of Information Science and Technology, Zhongkai University of Agriculture and Engineering, Guangzhou, 510225, China (e-mail: icken@126.com).

Fenglian Tie is with the School of Information Science and Technology, Zhongkai University of Agriculture and Engineering, Guangzhou, 510225, China (e-mail: tie2006x@163.com).

Haoran Huang is with the School of Information Science and Technology, Zhongkai University of Agriculture and Engineering, Guangzhou, 510225, China (e-mail: 93643764@qq.com).

Hanhui Lin is with the Center for Educational Technology, Guangdong University of Finance and Economics, Guangzhou 510320, China (e-mail: 65721450@qq.com).

Huazhou Chen (Corresponding author) is with the College of Science, Guilin University of Technology, Guilin, 541004, China (e-mail: huazhouchen@163.com).

This research was funded by the National Natural Science Foundation of China under Grant No.61505037, the National Spark Program under Grant No.2014GA780009, the State Scholarship Fund under Grant CSC No.201408440326, the Guangdong Natural Science Foundation under Grant No.S2013040014993, the Pearl River S\&T Nova Program of Guangzhou under Grant No.201506010035, the University-sponsored Research Project of Guangdong University of Finance and Economics under Grant No.14GLL63001, the Research Project of Research Institute of Education in Guangdong Province under Grant No.GDJY-2014-Cb043, the University Scientific Research Project of Guangxi Education Office under Grant No.KY2015ZL095. Submitted, 09 March 2015. Published as resubmitted by the authors on 24 August 2015. 